\title{
Stochastic Texture Analysis for Measuring Sheet Formation Variability in the Industry
}

\author{
Jacob Scharcanski, Senior Member, IEEE
}

\begin{abstract}
Several continuous manufacturing processes use stochastic texture images for quality control and monitoring. Large amounts of pictorial data are acquired, providing important information about both the materials produced and the manufacturing processes involved. However, it is often difficult to measure objectively the similarity among such industrial stochastic images or to discriminate between the texture images of stochastic materials with distinct properties. Nowadays, the degree of discrimination required by industrial processes often goes beyond the limits of human visual perception. This paper proposes a new approach for multiresolution stochastic texture discrimination in the industry (e.g., nonwoven textiles and paper), which is focused on sheet formation properties. The wavelet transform is used to represent stochastic texture images in multiple resolutions and to describe them using local density variability as features. At each resolution, the wavelet subbands approximate image gradients. The image gradients are modeled as Gaussian colored noise, and the gradient magnitudes, as Rayleigh probability density functions. Based on this representation, a multiresolution distance measure for stochastic textures is proposed. Some experimental results are reported, and ideas for future work are presented with the conclusions.
\end{abstract}

Index Terms-Gaussian colored noise, industrial quality control, maintenance, nonwoven textiles, Rayleigh, stochastic textures, wavelets.

\section{INTRODUCTION}

$\mathbf{I}$ N SEVERAL industrial continuous processes, static and dynamic stochastic texture images are acquired and used in quality control. In particular, stochastic texture images are used in the manufacture of foil-like materials such as nonwoven textiles, paper, polymer membranes, and conductor and semiconductor coatings. Important information can be extracted from these gray-level images, which represent the spatial density variations of such planar materials. In general, these images contain a composition of stochastic features at various scales, resulting from local clumping and aligning of the constituent matter with varying degree of regularity.

Sheet formation is an important parameter in the manufacture of stochastic planar materials. The spatial distribution of mass density, i.e., gray levels, is nearly random in the texture images obtained from these materials, and the gray-level autocorrela-

Manuscript received January 5, 2005; revised April 19, 2006. This work was supported by CNPq (Brazilian Research Council).

The author is with the Instituto de Informática, Universidade Federal do Rio Grande do Sul, Porto Alegre, Rio Grande do Sul 91501-970, Brazil (e-mail: jacobs@inf.ufrgs.br).

Digital Object Identifier 10.1109/TIM.2006.881036 tion tends to vary with the scale of analysis. ${ }^{1}$ The concept of sheet formation was used to describe the uniformity of local mass density variation in the sheet plane. Therefore, better sheet formation implies more uniform local gray-level variation.

Nowadays, paper and nonwoven textiles are produced in wide sheets and at growing speeds. During production, product quality is evaluated constantly in different points of the industrial sheet formers. Particularly, sheet formation is estimated in the samples collected across the machine sheet forming section (i.e., forming web). Formation variability across the forming web is a common problem in paper and nonwoven textile manufacturing, which generally requires expensive equipment for diagnosis and control. Commercially available formation testers are sometimes used, but they tend to be considered expensive by most manufacturers. Therefore, low-cost methods for testing and comparing sheet formation are currently in demand and are challenging researchers [1]-[3].

Industrial machine operators often try to evaluate visually the (collected) sample stochastic textures and estimate the manufacturing process condition using their experience in the field. This empirical approach is subjective and prone to failure mainly because the human vision is limited in terms of its ability to distinguish between stochastic textures [1], [3], [4]. Despite advances in texture representation and classification over the past three decades [4]-[7], the problem of stochastic texture feature interpretation and classification remains a challenge for researchers [8] and for a large segment of the industry [3] mostly because in several practical situations, stochastic texture analysis and classification must outperform visual texture discrimination and match industrial needs.

A substantial amount of work has been done in texture analysis in the wavelet domain (e.g., focusing on textures found in nature, arts, or design). However, little attention has been given to industrial stochastic textures (e.g., textures of nonwoven textiles and paper, which essentially look like noise). Therefore, most of the methods were proposed for texture analysis in general but were not designed for the specific problem of stochastic texture analysis in the industry, where the texture visual appearance is similar to noise [6], [9], [10]. Some methods perform regular energy sampling in the wavelet domain (e.g., [11]), but regular patterns are rarely found in these textures due to their stochastic nature. In addition, the methods based on higher order statistics generally do not make explicit relevant

\footnotetext{
${ }^{1}$ During the sheet forming process, fibers clump and align with varying degree of correlation, depending on the process control parameters, such as forming web speed and turbulence.
} 
stochastic texture features, which are important to industrial applications (where process conditions are estimated based on specific texture parameters, e.g., [5], [12], [13]).

In this paper, industrial stochastic textures are modeled as colored noise. A low-cost multiresolution scheme for comparing industrial stochastic textures in terms of sheet formation is proposed. Sheet formation is characterized here by Rayleigh distributions, representing the occurrences of image gradient magnitudes in multiple resolutions. ${ }^{2}$ We begin by describing how we model industrial stochastic textures and how we use wavelets to measure the image gradients in multiple resolutions. Next, a multiresolution distance measure for stochastic textures is introduced. Finally, we present some experimental results and conclusions. Possible applications of the proposed method are in maintenance and quality control in the paper and nonwoven textiles industry.

\section{Multiple-Resolution Analysis of Industrial STOCHASTIC TEXTURES}

For a given thickness (i.e., average mass density), industrial materials such as nonwoven textiles and paper may be produced with different formations (i.e., different local mass variability properties). In this paper, local mass variability is represented by local image gradients of the sample texture images, which are calculated at multiple resolutions.

To estimate the local gradients in multiple resolutions, we apply the undecimated two-dimensional wavelet transform (WT) proposed by Mallat and Zhong [14]. This WT requires the calculation of two detail images using one smoothing function $\phi(x, y)$ and two wavelets $\psi^{i}(x, y)$. The dilation of these functions are denoted by [14]

$$
\begin{aligned}
\phi_{s}(x, y) & =\frac{1}{s^{2}} \phi\left(\frac{x}{s}, \frac{y}{s}\right) \\
\text { and } \quad \psi_{s}^{i}(x, y) & =\frac{1}{s^{2}} \psi^{i}\left(\frac{x}{s}, \frac{y}{s}\right), \quad i=1,2
\end{aligned}
$$

and the dyadic WT $f(x, y)$ at a scale $s=2^{j}$ has two detail components given by

$$
W_{2^{j}}^{i} f(x, y)=\left(f * \psi_{2^{j}}^{i}\right)(x, y), \quad i=1,2
$$

and one low-pass component given by

$$
S_{2^{j}} f(x, y)=\left(f * \phi_{2^{j}}\right)(x, y) .
$$

The coefficients $W_{2^{j}}^{1} f(x, y)$ and $W_{2^{j}}^{2} f(x, y)$ represent the details in the $x$ and $y$ directions, respectively. Thus, the image gradient at resolution $2^{j}$ can be approximated by [14], [15]

$$
\mathbf{W}_{2^{j}} f(x, y)=\left(\begin{array}{l}
W_{2^{j}}^{1} f(x, y) \\
W_{2^{j}}^{2} f(x, y)
\end{array}\right)
$$

where $j=1,2, \ldots, J$.

Since we are dealing with digital images $f[n, m]$, we use the discrete version of the WT [14], and the discrete

\footnotetext{
${ }^{2}$ Image gradients represent the local variation of gray levels in multiple resolutions.
}

wavelet coefficients are denoted in this paper by $W_{2^{j}}^{i} f[n, m]$, for $i=1,2$.

Industrial stochastic textures, such as nonwoven textiles and paper, are textures characterized by near-random gray-level spatial variability, which often present spatial correlations that persist over short, medium, or long ranges. The stochastic nature and correlation patterns of these textures originate from the stochastic fiber entanglements and alignments that take place during the nonwoven and paper forming process [1], [3].

It can be shown that the gray-level (i.e., local density) distributions in these textures can be modeled as a Gaussian process, with some deviation from normality at the end of the distribution tails. Therefore, the input texture image $f(x, y)$ is modeled as Gaussian colored noise $\epsilon(x, y)$, and we assume that it is an ergodic stationary process, which is characterized by its mean and spatial correlation at each scale $j$ [16]. Since the WT is a linear transform, the dyadic WT $\epsilon(x, y)$ or the WT $\epsilon$ (using a simpler notation) at a scale of $s=2^{j}$ has two detail components given by

$$
U_{j}^{i}=W_{2^{j}}^{i} \epsilon=\left(\epsilon * \psi_{2^{j}}^{i}\right), \quad i=1,2
$$

where the $W_{2^{j}}^{i} \epsilon \mathrm{S}$ are also modeled as Gaussian colored noise processes, with their standard deviations being $\sigma_{U_{j}}$. The indexes $i=1,2$ correspond to the $x$ and $y$ directions, respectively.

Consequently, the occurrences of the coefficients $W_{2^{j}}^{1} \epsilon[n, m]$ and $W_{2^{j}}^{2} \epsilon[n, m]$ are approximated by Gaussian distributions. The normal plots in Fig. 1(c) and (d) show that the Gaussian model can represent a wide range of wavelet coefficient values in stochastic textures, with some deviation from Gaussian occurring in the tails of the distribution. This experimental evidence was confirmed in a large set of samples.

Thus, the corresponding distribution of magnitudes $M_{2^{j}} f=$ $\left[\left(W_{2^{j}}^{1} f\right)^{2}+\left(W_{2^{j}}^{2} f\right)^{2}\right]^{1 / 2}$, which is denoted simply by $M_{2^{j}}$, may be approximated by a Rayleigh probability density function (pdf) [17], i.e.,

$$
R_{j}\left(M_{2^{j}}\right)=\frac{M_{2^{j}}}{\left[b^{j}\right]^{2}} e^{\frac{-M_{2 j}^{2}}{\left[2 b^{j}\right]^{2}}}
$$

where

$$
b^{j}=\frac{\sqrt{2 \sigma_{M_{2 j}}^{2}}}{4-\pi}
$$

and $\sigma_{M_{2 j}}$ is the standard deviation of the gradient magnitudes at scale $2^{j}$, for $j=1,2, \ldots, J$.

Fig. 1(e) and (f) illustrates the Rayleigh model fitting the actual gradient magnitude distribution.

The image gray levels are associated with the local mass density, and the gray-level gradient magnitudes are associated with the local mass variation. Let us assume gray levels that are distributed spatially as an ergodic stationary Gaussian colored noise process. In this case, small image gradients imply smooth transitions between the local maxima and minima and small local mass variation. Good formation occurs when the stochastic gradient magnitudes are small in average and the standard 


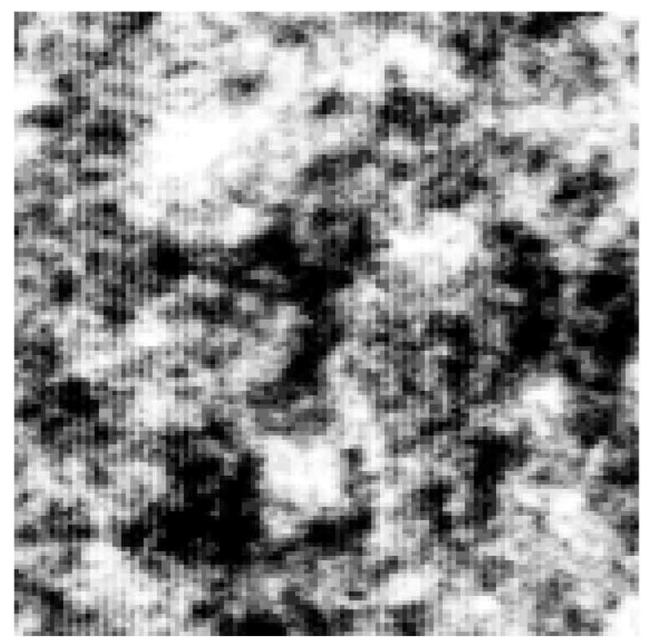

(a)

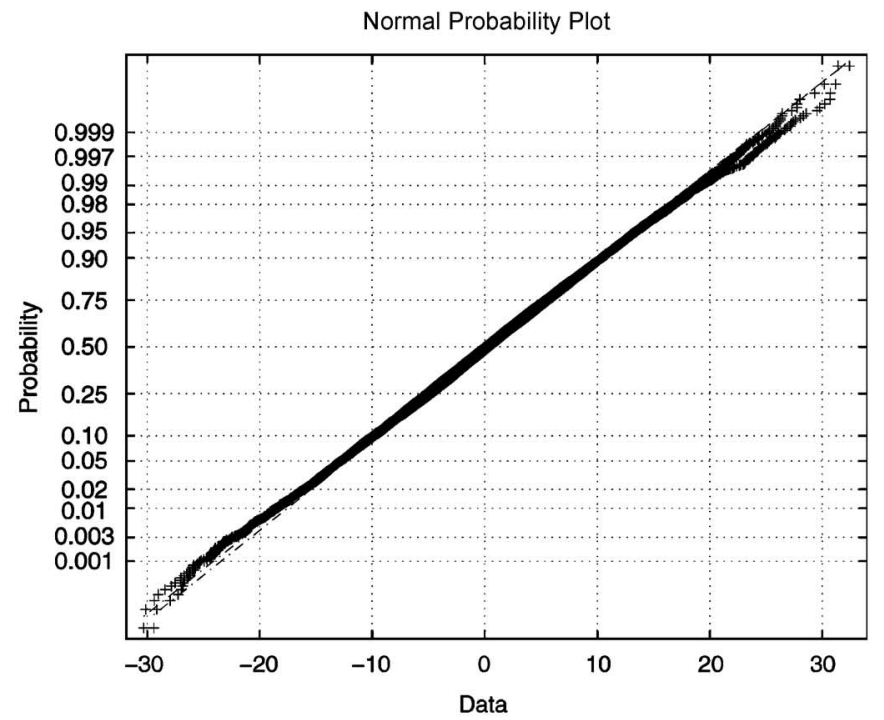

(c)

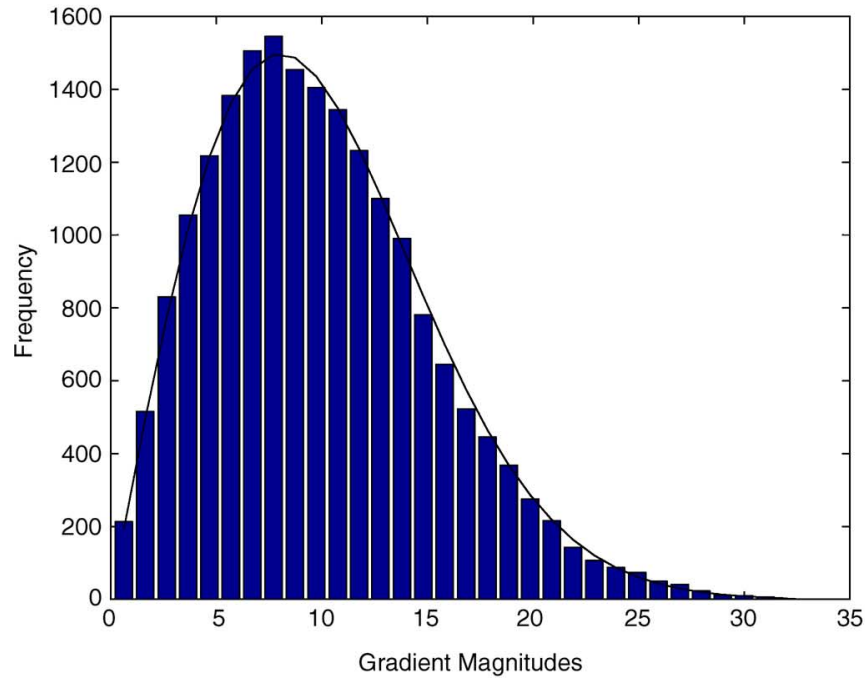

(e)

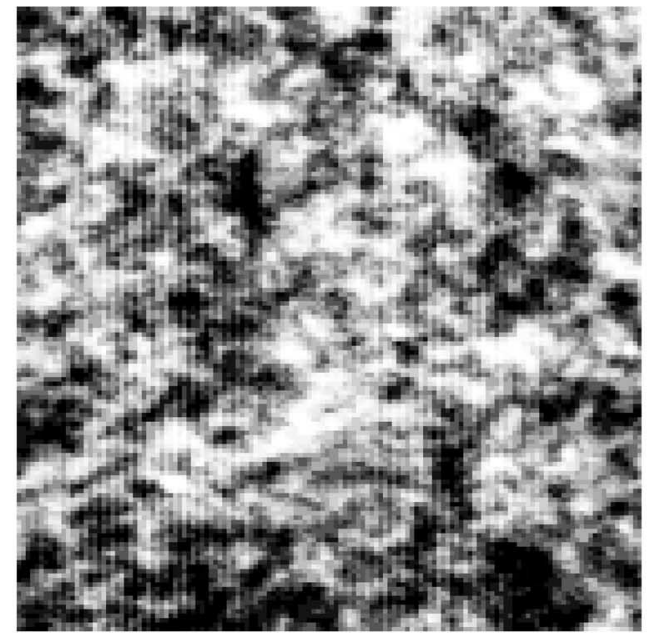

(b)

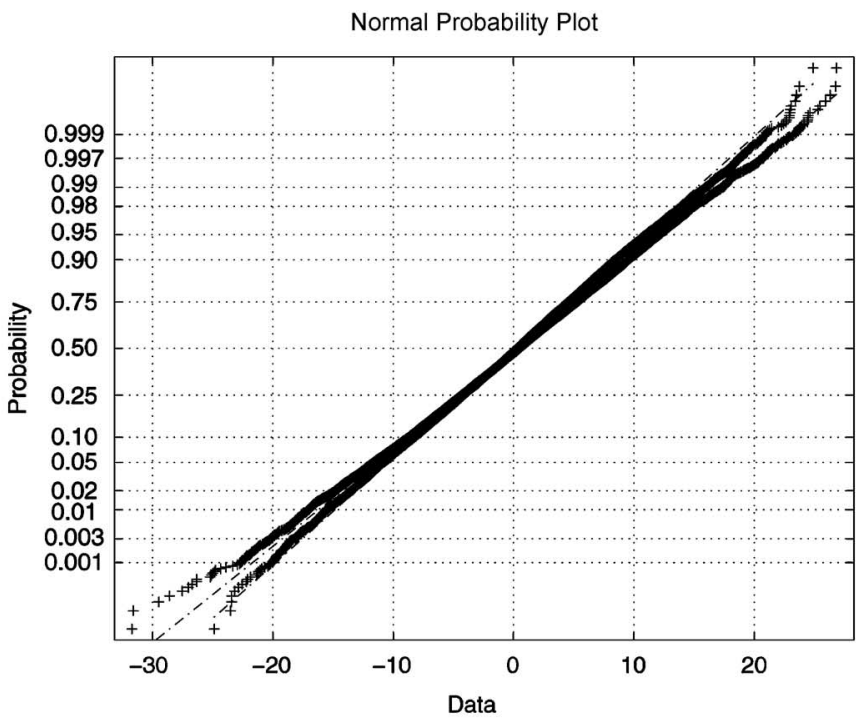

(d)

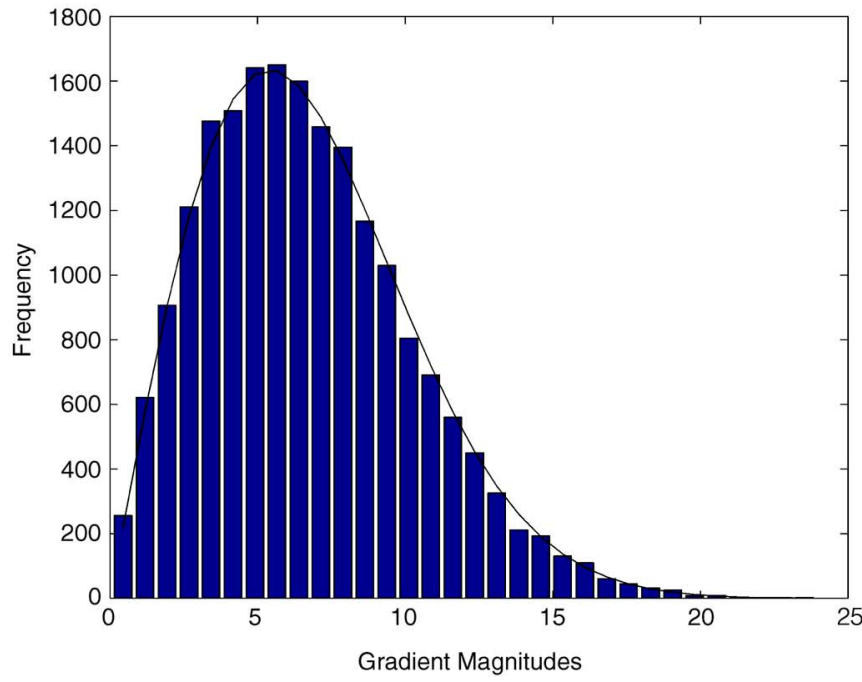

(f)

Fig. 1. Comparative results for paper and nonwoven textiles sample images ( $\beta$ radiographs): (a) sample 1 and (b) sample 2 . Normal plots of coefficients $W^{i}$, $i=1,2$ (middle scale $j=2$ ): (c) sample 1 and (d) sample 2. Gradient magnitude distributions (Rayleigh pdf fit: solid): (e) sample 1 (f) sample 2 (histogram equalization was applied to the images in display to improve their visualization). (Color version available online at http://ieeexplore.ieee.org.) 


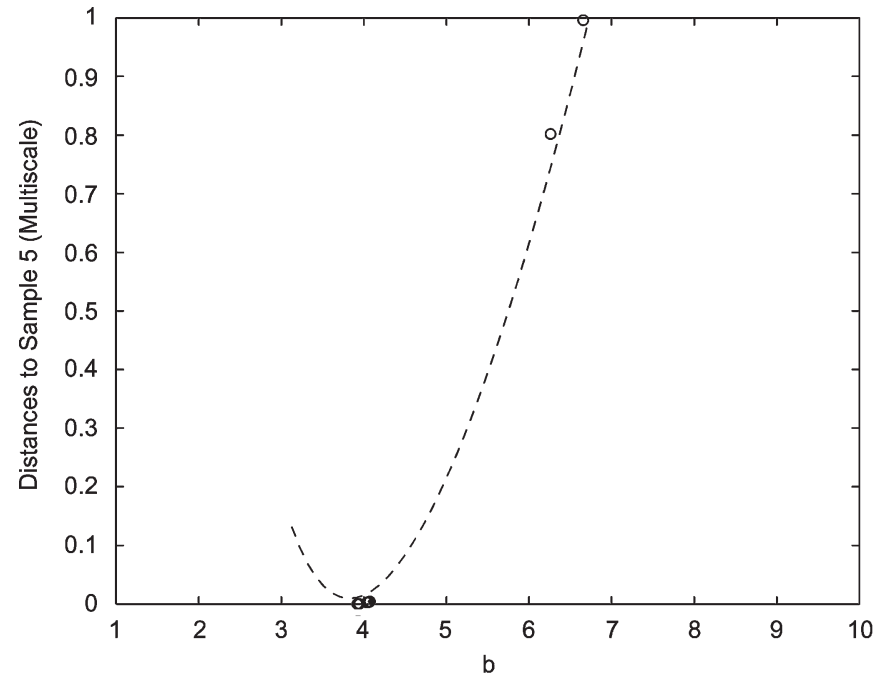

(a)

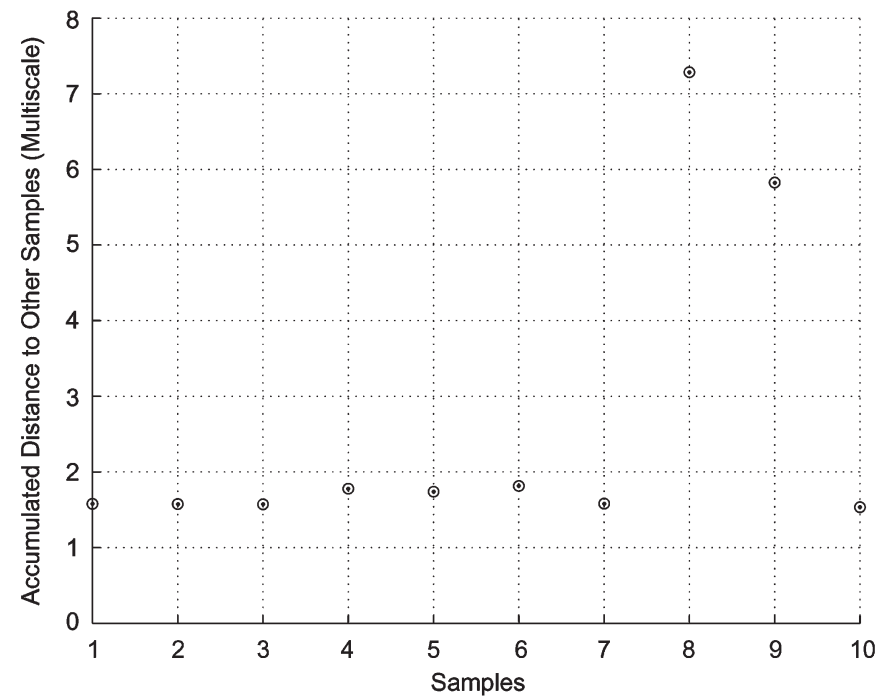

(c)

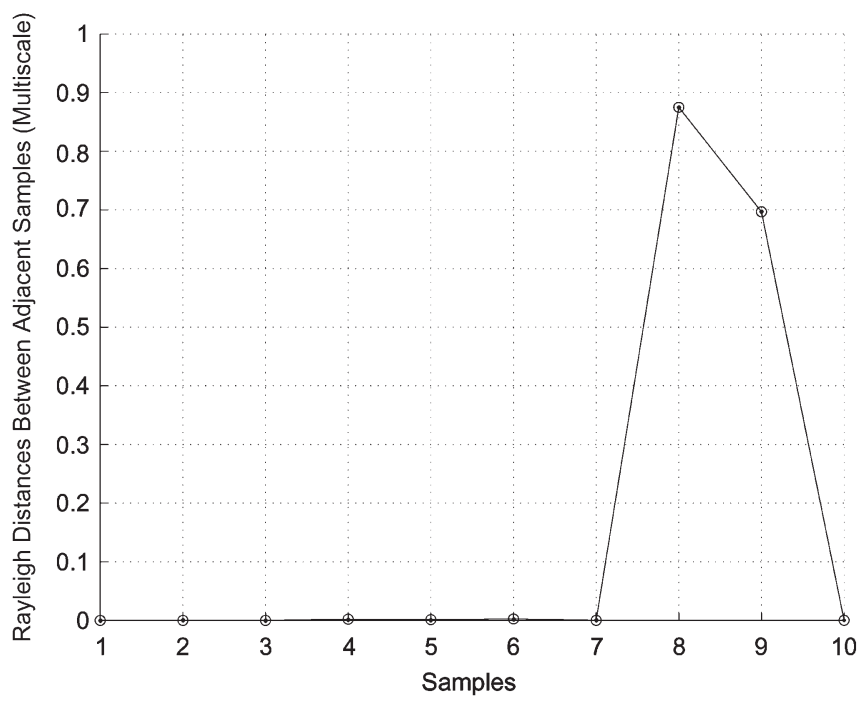

(e)

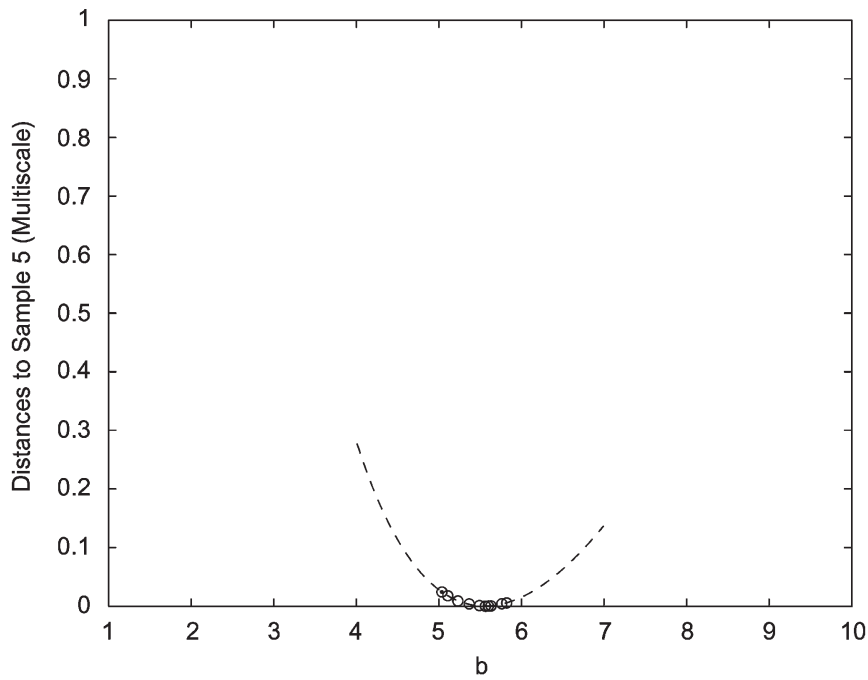

(b)

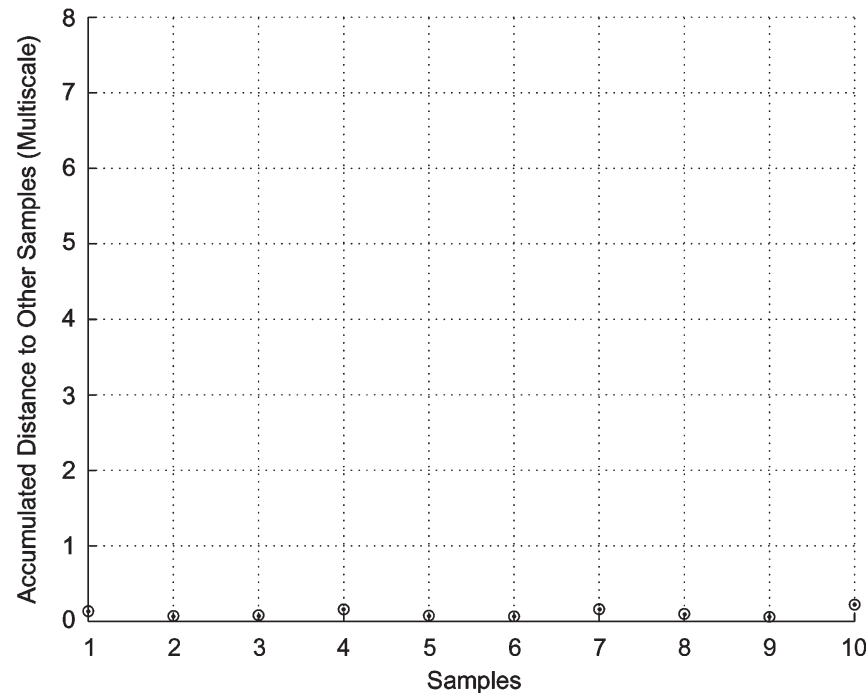

(d)

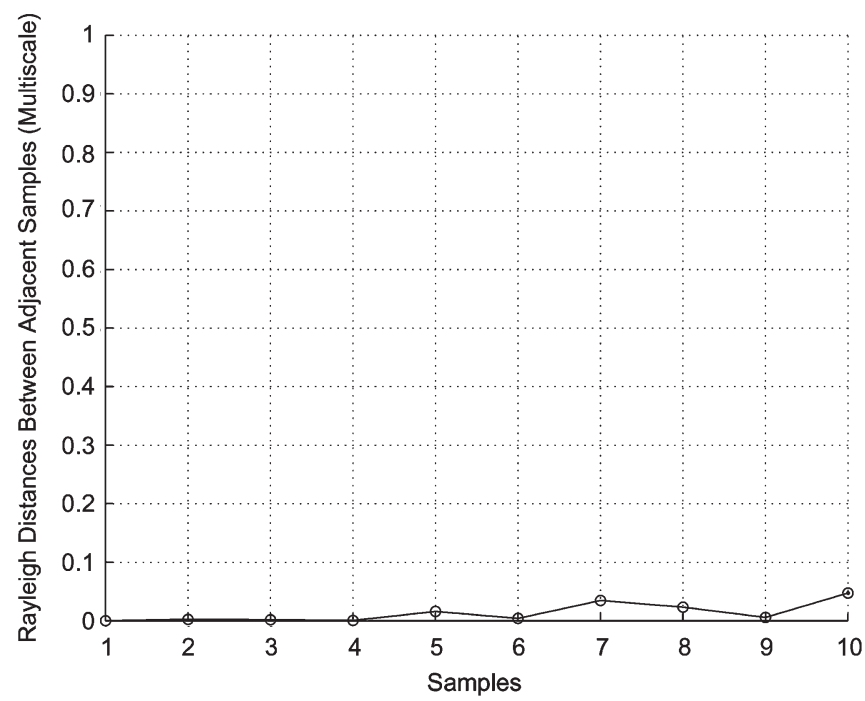

(f)

Fig. 2. Tests to evaluate sheet formation along the CD. Distances to the sample located at the center of the web (i.e., sample 5). Circle/dots are samples, and the $D_{\text {Rayl }}{ }^{s}$ space in the vicinity of sample 5 is indicated by the dotted line: (a) nonstandard operating conditions and (b) standard operating conditions. Accumulated distance $D_{k}^{\text {acc }}$ to sample $k$ : (c) web profile for nonstandard operating conditions and (d) web profile for standard operating conditions. Distances $D_{\mathrm{Rayl}}{ }^{s},(R(b k)$, $R(b k+1)$ ) between the samples adjacently located on the web: (e) web profile for nonstandard operating conditions and (f) web profile for standard operating conditions. 
deviation of gradient magnitudes is also small (i.e., the local mass variation is relatively uniform). At a scale $j$, these stochastic texture characteristics are modeled by a Rayleigh distribution with parameter $b^{j}$ and mean and standard deviation given by $\mu_{M_{2 j}}=b^{j} \sqrt{\pi / 2}$ and $\sigma_{M_{2 j}}=\left(\left(b^{j}\right)^{2}(4-\pi)\right) / 2$, respectively. If the distribution of $M_{2^{j}}$ values presents a small $b^{j}$, then $\mu_{M_{2 j}}$ and $\sigma_{M_{2 j}}$ are also small, indicating good formation. A worse formation condition is indicated by larger values of $b^{j}, \mu_{M_{2^{j}}}$, and $\sigma_{M_{2^{j}}}$. Typically, samples of textures with similar formation also have similar $b^{j}$ parameters, and this information may be used for comparing the formation in industrial stochastic textures.

\section{Similarity MEASURE FOR COMPARING INDUSTRIAL STOCHASTIC TEXTURES}

Two industrial stochastic textures can be compared in terms of formation using the Kullback-Leibler distance between their Rayleigh distributions $R\left(b_{1}\right)$ and $R\left(b_{2}\right)$, i.e.,

$$
D_{\text {Rayl }}\left(R\left(b_{1}\right), R\left(b_{2}\right)\right)=-\frac{1}{\pi} \log \left(\frac{b_{2}}{b_{1}}-\frac{b_{2}^{2}}{b_{1}^{2}}+1\right)
$$

where $b_{1}$ and $b_{2}$ are the Rayleigh parameters of the textures, respectively.

In this paper, we use a symmetrical version of the Kullback-Leibler distance, i.e.,

$$
D_{\text {Rayl }}{ }^{s}=D_{\text {Rayl }}\left(R\left(b_{1}\right), R\left(b_{2}\right)\right)+D_{\text {Rayl }}\left(R\left(b_{2}\right), R\left(b_{1}\right)\right) .
$$

The Kullback-Leibler distance has desirable properties. Its convexity guarantees that a minimum exists, and in order to calculate the Kullback-Leibler distance from multiple scales, i.e., feature sets representing different wavelet subbands, we can use the "chain rule" [18] such that

$$
p\left(V_{1}, V_{2}, \ldots, V_{n}\right)=p\left(V_{1} \mid V_{2} \cdots V_{n}\right) \cdots p\left(V_{n}\right)
$$

where $p\left(V_{1}, V_{2}, \ldots, V_{n}\right)$ is a joint pdf with $n$ marginal pdfs $p\left(V_{1}\right), \ldots, p\left(V_{n}\right)$. The Kullback-Leibler distance between two joint pdfs $p^{a}$ and $p^{b}$ is given by

$$
\begin{aligned}
D & \left(p^{a}\left(V_{1}, V_{2}, \ldots, V_{n}\right), p^{b}\left(V_{1}, V_{2}, \ldots, V_{n}\right)\right) \\
= & D\left(p^{a}\left(V_{1} \mid V_{2} \cdots V_{n}\right), p^{b}\left(V_{1} \mid V_{2} \cdots V_{n}\right)\right)+\cdots \\
& \quad+D\left(p^{a}\left(V_{n-1} \mid V_{n}\right), p^{b}\left(V_{n-1} \mid V_{n}\right)\right)+D\left(p^{a}\left(V_{n}\right), p^{b}\left(V_{n}\right)\right)
\end{aligned}
$$

and it can be verified that

$$
\begin{gathered}
D\left(p^{a}\left(V_{1}, V_{2}, \ldots, V_{n}\right), p^{b}\left(V_{1}, V_{2}, \ldots, V_{n}\right)\right) \\
\geq \frac{1}{n}\left[D\left(p^{a}\left(V_{1}\right), p^{b}\left(V_{1}\right)\right)+D\left(p^{a}\left(V_{2}\right), p^{b}\left(V_{2}\right)\right)\right. \\
\left.+\cdots+D\left(p^{a}\left(V_{n}\right), p^{b}\left(V_{n}\right)\right)\right] .
\end{gathered}
$$

Introducing the symmetrical version of the Kullback-Leibler distance in (11) and (12) yields

$$
\begin{aligned}
D^{s} & \left(p^{a}\left(V_{1}, V_{2}, \ldots, V_{n}\right), p^{b}\left(V_{1}, V_{2}, \ldots, V_{n}\right)\right) \\
= & D\left(p^{a}\left(V_{1}, V_{2}, \ldots, V_{n}\right), p^{b}\left(V_{1}, V_{2}, \ldots, V_{n}\right)\right) \\
& \quad+D\left(p^{b}\left(V_{1}, V_{2}, \ldots, V_{n}\right), p^{a}\left(V_{1}, V_{2}, \ldots, V_{n}\right)\right)
\end{aligned}
$$

and

$$
\begin{aligned}
D^{s}\left(p^{a}\left(V_{1}, V_{2}, \ldots, V_{n}\right), p^{b}\left(V_{1}, V_{2}, \ldots, V_{n}\right)\right) \\
\geq \frac{1}{n}\left[D\left(p^{a}\left(V_{1}\right), p^{b}\left(V_{1}\right)\right)+D\left(p^{b}\left(V_{1}\right), p^{a}\left(V_{1}\right)\right)\right. \\
\quad+D\left(p^{a}\left(V_{2}\right), p^{b}\left(V_{2}\right)\right)+D\left(p^{b}\left(V_{2}\right), p^{a}\left(V_{2}\right)\right)+\cdots \\
\left.\quad+D\left(p^{a}\left(V_{n}\right), p^{b}\left(V_{n}\right)\right)+D\left(p^{b}\left(V_{n}\right), p^{a}\left(V_{n}\right)\right)\right]
\end{aligned}
$$

respectively.

Therefore, the Kullback-Leibler distance between two joint pdfs has the average distance between marginals as the lower bound. This distance lower bound provides a feature space contractive mapping and can be interpreted as a simpler version of the "full distance" shown in (13). The distance lower bound provides a simpler distance measure for sample classification and guarantees no false dismissals [19].

It can be observed that $V_{1}, V_{2}, \ldots, V_{n}$ are feature sets representing different wavelet subbands. If the same $n$ subbands are used to discriminate between samples, the factor $1 / n$ is a multiplying constant that affects all sample-to-sample distances equally and may be dropped without affecting the results. In this case, the joint Kullback-Leibler distance is approximated simply by the sum of $n$ subband feature set distances, i.e.,

$$
\begin{aligned}
D^{s} & \left(p^{a}\left(V_{1}, V_{2}, \ldots, V_{n}\right), p^{b}\left(V_{1}, V_{2}, \ldots, V_{n}\right)\right) \\
& \simeq \sum_{i=1, \ldots, n} D\left(p^{a}\left(V_{i}\right), p^{b}\left(V_{i}\right)\right)+D\left(p^{b}\left(V_{i}\right), p^{a}\left(V_{i}\right)\right) .
\end{aligned}
$$

Recalling that in this paper, marginals are Rayleigh distributions $R\left(b_{i}\right)$, the proposed distance measure is

$$
\begin{aligned}
& D_{\text {Rayl }^{s}}\left(R^{a}\left(b_{1}, b_{2}, \ldots, b_{n}\right), R^{b}\left(b_{1}, b_{2}, \ldots, b_{n}\right)\right) \\
& \simeq \sum_{i=1, \ldots, n} D_{\text {Rayl }}\left(R^{a}\left(b_{i}\right), R^{b}\left(b_{i}\right)\right)+D_{\text {Rayl }}\left(R^{b}\left(b_{i}\right), R^{a}\left(b_{i}\right)\right)
\end{aligned}
$$

where $i$ corresponds to scale $j$ and $n=J$.

In the next section, we will discuss the efficacy of our stochastic texture distance measure based on experimental results.

\section{EXPERIMENTAL RESULTS}

The manufacturing process of nonwoven textiles and paper has its stochastic dynamics influenced by the process 
parameters (e.g., web speed and turbulence). Due to fluctuations and irregularities in the manufacturing process, there are corresponding variations in the makeup of fibers and the orientation of fibers across the moving web [i.e., along the direction orthogonal to the moving web (CD) and the moving web direction or yet, the machine direction (MD)] [20]. Consequently, the physical properties, such as sheet formation, may vary in both the MD (machine/web direction) and the CD (machine/web cross direction). To evaluate how homogeneous (or heterogeneous) the sheet physical properties are, it is common to test the samples collected at several locations along the CD of a given machine. Under normal operating conditions, sheet formation is relatively homogeneous across the machine profile (i.e., the $\mathrm{CD}$ ). However, if sheet formation presents significant variability along the $\mathrm{CD}$, parameter adjustments or maintenance may be required.

Next, the performance of our approach is illustrated using two sets of ten samples equally spaced along the manufacturing web CD (i.e., $15 \mathrm{~cm}^{2}$ each and spaced $50 \mathrm{~cm}$ from each other). One of the sample sets represents standard operating conditions, and the other sample set represents a deviation from the standard operating conditions (i.e., these were obtained before stopping production for maintenance). The central part of these samples were scanned using a scanner with a transparency unit that obtains images with $1000 \times 1100$ pixels at $600 \mathrm{dpi}$. Three consecutive dyadic scales were used $\left(2^{j}\right.$, for $\left.j=1,2,3\right)$ in the texture analysis. These experiments were conducted to evaluate sheet formation uniformity along the $\mathrm{CD}$, as illustrated in Fig. 2(a) and (b). These results indicate that we can discriminate standard from nonstandard operating conditions considering the $D_{\text {Rayl }^{s}}$ distances obtained with respect to the sample located at the center of the web. Formation homogeneity is characterized by the small variability across the web. Consequently, sheet formation is more uniform under standard operating conditions (i.e., has smaller distance coefficient of variation of 1.15) than under nonstandard operating conditions (i.e., the distance coefficient of variation is 1.47).

The aforementioned tests indicate whether sheet formation is homogeneous along the $\mathrm{CD}$. However, they do not indicate the locations where the moving web is more homogeneous/heterogeneous. Given a set $\mathbf{S}$ of $k$ samples along the $\mathbf{C D}$, the sum of pairwise distances between each sample $k(\epsilon \mathbf{S})$ and the remaining $k-1$ samples in $\mathbf{S}$ (namely $D_{k}^{\text {acc }}$ ) can be used as a $\mathrm{CD}$ sheet formation homogeneity indicator, i.e.,

$$
\begin{aligned}
D_{k}^{\text {acc }}=\sum_{p=1}^{\sharp S} \sum_{j=1}^{J} D_{\text {Rayl }}\left(R^{k}\left(b_{j}\right), R^{p}\left(b_{j}\right)\right) & \\
& +D_{\text {Rayl }}\left(R^{p}\left(b_{j}\right), R^{k}\left(b_{j}\right)\right) .
\end{aligned}
$$

Fig. 2(c) and (d) shows that different $D_{k}^{\text {acc }}$ profiles are obtained for different operating conditions. Under regular operating conditions, the $\mathrm{CD} D_{k}^{\text {acc }}$ profile shows smaller variability across the web-even at web extremities, where higher variability is expected; on the other hand, for nonstandard operating conditions, higher $D_{k}^{\text {acc }}$ values and variability are obtained, as
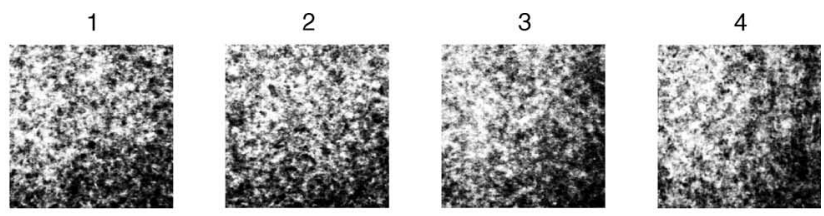

5
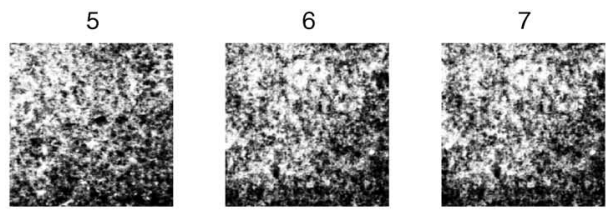

(a)

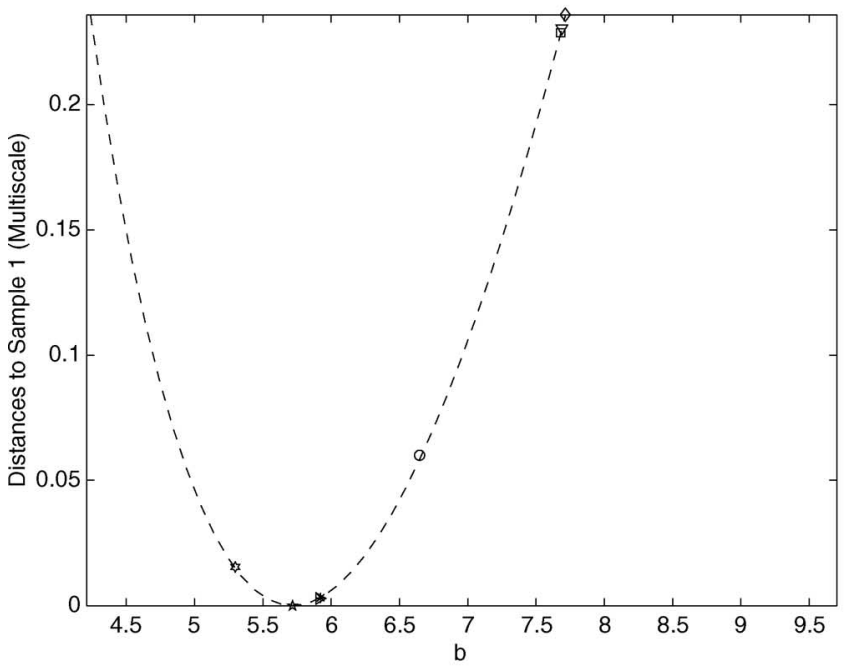

(b)

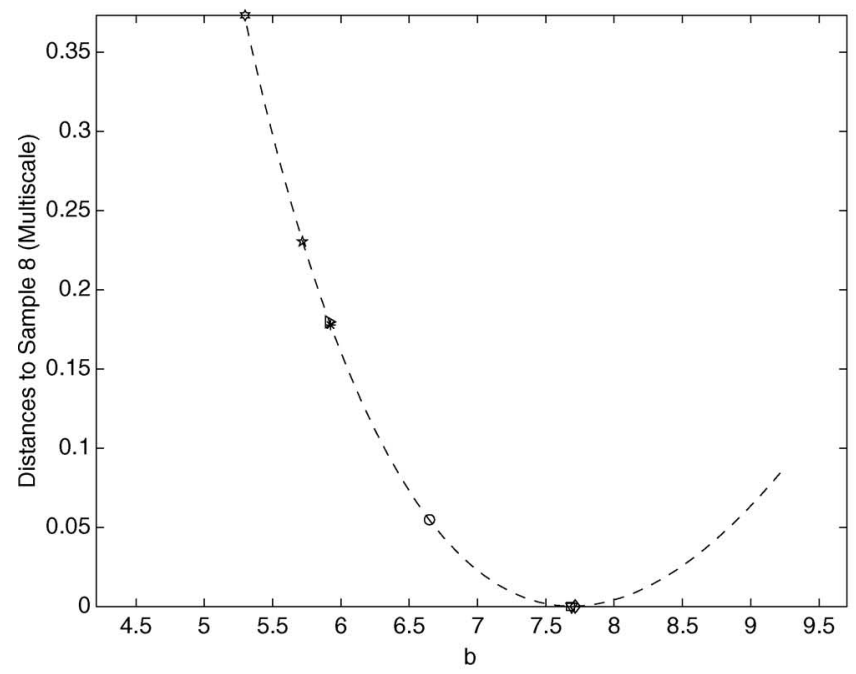

(c)

Fig. 3. Tests to evaluate the distance measurements using sheets with known formation. The $D_{\text {Rayls }}$ space in the vicinity of the sample of interest is indicated by the dotted line, and sample notations are indicated in Table I. (a) Sample images of the eight stochastic textures used in the experiments (these are $\beta$-radiographs; histogram equalization was applied to the images in display to improve their visualization). Distances $D_{\mathrm{Rayl}^{s}}$ to selected samples: (b) distances to sample 1 and (c) distances to sample 8.

illustrated in Fig. 2(c) - particularly, at the right side of the moving web, where variability is higher. It should be noticed that this web homogeneity test compares stochastic texture 
TABLE I

SAMPLES With THE KNOWN MEASUREd Formation Numbers [21] Used IN THE Tests. These Eight SAMPles Form ThreE GRoups: Small Formation Numbers (i.e., SAmples 1, 2, 3, AND 4), MEDiUM Formation Number (SAMPLE 5), AND HIGH FORMATION NUMBERS (SAMPLES 6,7 , AND 8)

\begin{tabular}{|l|c|c|c|}
\hline Sample & Name [21] & Formation Number (1 mm) & Notation \\
\hline 1 & HSMA33B1 & 2.42 & pentagram \\
\hline 2 & HSMA33B3 & 2.20 & triangle (right) \\
\hline 3 & HXXN32G1 & 2.03 & hexagram \\
\hline 4 & HSMA33B2 & 2.28 & asterisk \\
\hline 5 & MGA695A4 & 5.23 & circle \\
\hline 6 & MBMC50A3 & 7.30 & square \\
\hline 7 & MBMC50A7 & 7.29 & diamond \\
\hline 8 & MBMC50A6 & 7.07 & triangle (down) \\
\hline
\end{tabular}

images and only requires that the imaging conditions remain the same. Similar conclusions may be derived from Fig. 2(e) and (f), where the distances between adjacent samples on the web are plotted as a profile. As expected, higher variability is found under nonstandard operating conditions at the right side of the moving web [Fig. 2(e)].

To confirm whether the proposed method is capable of sorting (clustering) samples based on their formation, a set of eight samples with known sheet formations were tested. These samples were selected from a standard industrial image database of nonwoven textiles and paper [21]. We chose samples from different forming machines, with different furnishes and grammages. ${ }^{3}$ All these images have a resolution of $140 \times 140$ pixels, with a spatial resolution of $0.2 \times 0.2 \mathrm{~mm}^{2} /$ pixel [21]. Fig. 3(a) shows the sample images. Table I shows the measured formation numbers [21] and indicates the sample notations in Fig. 3(b) and (c). Samples 1, 2, 3, and 4 have smaller formation numbers and have clustered close to sample 1 , as shown in Fig. 3(b). On the other hand, samples 6, 7, and 8 , which have higher formation numbers, clustered close to sample 8, as shown in Fig. 3(c). As expected, sample 4 has a medium formation number and stayed halfway between the groups of samples with higher and smaller formation numbers. Therefore, the proposed method sorts samples based on their formation, which is in agreement with the measured formation numbers.

\section{CONCluding Remarks}

In summary, stochastic texture images are acquired in large quantities in continuous industrial processes and encode important quality and process information. Moreover, the tex-

\footnotetext{
${ }^{3}$ Grammage is defined as the mass density in gram per square meter.
}

ture analysis methods required by some industrial processes must be objective because texture discrimination often exceeds the limits of human visual perception. Therefore, analyzing and discriminating industrial stochastic textures are challenging tasks.

The preliminary experimental results obtained by our approach were encouraging. Our proposed stochastic texture representation and distance can lead to new low-cost methods for the evaluation of production machine calibrations. In addition, the experiments indicate that our approach can help in discriminating (i.e., sorting or clustering) samples based on their formation, and the obtained results tend to agree with formation number measurements. However, more extensive testing is needed to achieve high confidence levels in noncontact methods for obtaining formation estimates, which are similar to those obtained in physical laboratory tests.

As future work, we intend to study the influence of different scale weighting schemes and use our approach as support for preventive maintenance and personnel training.

\section{ACKNOWLEDGMENT}

The author would like to thank O. Machado of Aracruz Celulose, Brazil, for providing the experimental data; Prof. W. Sampson of the Material Sciences Department, UMIST, U.K., for helping with sample formation measurements; Prof. R. da Silva of the Instituto de Informática, UFRGS, Brazil; and Prof. J. A. Barrionuevo and Prof. S. R. Lopes of the Instituto de Matemática, UFRGS, Brazil, for their advice and useful discussions.

\section{REFERENCES}

[1] M. Deng and C. T. J. Dodson, Paper: An Engineered Stochastic Structure. Atlanta, GA: TAPPI Press, 1994.

[2] X. Z. Wang, Data Mining and Knowledge Discovery for Process Monitoring and Control. London, U.K.: Springer-Verlag, 1999.

[3] J. Scharcanski and C. T. J. Dodson, "Local spatial anisotropy and its variability," IEEE Trans. Instrum. Meas., vol. 49, no. 5, pp. 971-979, Oct. 2000.

[4] Y. Rui, T. S. Huang, and S.-F. Chang, "Image retrieval: Past, present and future," J. Vis. Commun. Image Represent., vol. 10, no. 1, pp. 1-23, 1999.

[5] G. Fan and X. Xia, "Wavelet-based texture analysis and synthesis using hidden Markov models," IEEE Trans. Circuits Syst. I, Fundam. Theory Appl., vol. 50, no. 1, pp. 106-120, Jan. 2003.

[6] S. Arivazhagan and L. Ganesan, "Texture classification using wavelet transform,” Pattern Recognit. Lett., vol. 24, no. 9/10, pp. 1513-1521, Jun. 2003.

[7] S. W. Baik and P. W. Pachowicz, "Online model classification for adaptive texture recognition in image sequences," IEEE Trans. Syst., Man, Cybern. A, Syst., Humans, vol. 32, no. 6, pp. 625-639, Nov. 2002.

[8] S. C. Zhu, Y. Wu, and D. Mumford, "Filters, random fields and maximum entropy," Int. J. Comput. Vis., vol. 27, no. 2, pp. 1-20, 1998.

[9] R. Manthalkar, P. K. Biswas, and B. N. Chatterji, "Rotation and scale invariant texture features using discrete wavelet packet transform," Pattern Recognit. Lett., vol. 24, no. 14, pp. 2455-2462, Oct. 2003.

[10] M. N. Do and M. Vetterli, "Wavelet-based texture retrieval using generalized Gaussian density and Kullback-Leibler distance," IEEE Trans. Image Process., vol. 11, no. 2, pp. 146-158, Feb. 2002.

[11] N. Kim and S. Udpa, "Texture classification using rotated wavelet filters," IEEE Trans. Syst., Man, Cybern. A, Syst., Humans, vol. 30, no. 6, pp. 847-852, Nov. 2000.

[12] H. Choi and R. G. Baraniuk, "Multiscale image segmentation using wavelet-domain hidden Markov models," IEEE Trans. Image Process., vol. 10, no. 9, pp. 1309-1321, Sep. 2001. 
[13] J. K. Romberg, H. Choi, and R. G. Baraniuk, "Bayesian tree structured image modeling using wavelet-domain hidden Markov models," IEEE Trans. Image Process., vol. 10, no. 7, pp. 1056-1068, Jul. 2001.

[14] S. G. Mallat and S. Zhong, "Characterization of signals from multiscale edges," IEEE Trans. Pattern Anal. Mach. Intell., vol. 14, no. 7, pp. 710-732, Jul. 1992.

[15] J. Scharcanski, C. R. Jung, and R. T. Clarke, "Adaptive image denoising using scale-space consistency," IEEE Trans. Image Process., vol. 11, no. 9, pp. 1092-1101, Sep. 2002.

[16] G. M. Jenkins and T. G. Watts, Spectral Analysis and its Applications. San Francisco, CA: Holden-Day, 1968.

[17] H. J. Larson and B. O. Shubert, Probabilistic Models in Engineering Sciences, vol. I. New York: Wiley, 1979.

[18] A. Papoulis, Probability, Random Variables and Stochastic Processes. New York: McGraw-Hill, 1991.

[19] M. Ranganathan, C. Faloutsos, and Y. Manolopoulos, "Fast subsequence matching in time-series databases," in Proc. ACM SIGMOD, 1994, pp. 419-429.

[20] G. A. Smook, Handbook for Pulp and Paper Technologists, 2nd ed. Vancouver, BC, Canada: Angus Wilde Publ., 1994.

[21] C. Dodson, W. K. Ng, and R. R. Singh, Paper Stochastic Structure Analysis-Archive 2 (CD-ROM). Toronto, ON, CA: Univ. Toronto, 1995.

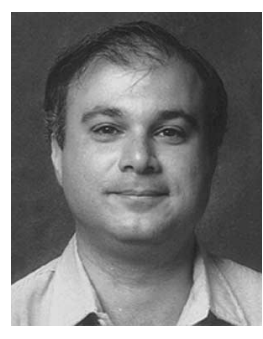

Jacob Scharcanski (M'03-SM'03) received the B.Eng. degree in electrical engineering and the M.Sc. degree in computer science from the Federal University of Rio Grande do Sul, Porto Alegre, RS, Brazil, in 1981 and 1984, respectively, and the Ph.D. degree in systems design engineering from the University of Waterloo, Waterloo, ON, Canada, in 1993.

He was a Postdoctoral Fellow with the Pulp and Paper Centre, University of Toronto, Toronto, ON, working on stochastic texture analysis and interpretation for industrial automation in the pulp and paper industry. He was also a Postdoctoral Fellow with the Communications Group, Department of Electrical and Computer Engineering, University of Toronto, working on image processing and analysis. He is currently an Associate Professor with the Institute of Informatics, Federal University of Rio Grande do Sul. He is the author and coauthor of more than 75 publications in journals and conferences and is the holder of two pending patents. His research interests include image processing and analysis, machine learning, and pattern recognition.

Prof. Scharcanski is a Professional Engineer (Professional Engineers Ontario, Canada, and Regional Council of Engineering and Architecture of Rio Grande do Sul, Brazil) and a member of the Brazilian Computer Society. 